\title{
Diagnósticos e percepções de uma formação docente sobre simulação realística de alta fidelidade na área da saúde
}

\author{
Diagnostics and perceptions of a teaching education on high fidelity realistic simulation \\ in the health area
}

\author{
Marcelo Taglietti ${ }^{*}$, Adriana Zilly², Clodis Boscarioli³ \\ ${ }^{1}$ Doutor em Educação Física, Professor Colaborador da Universidade Estadual do Oeste do Paraná (UNIOESTE); \\ ${ }^{2}$ Doutora em Ciências Biológicas, Professora Associada da UNIOESTE; \\ ${ }^{3}$ Doutor em Engenharia Elétrica, Professor Associado da UNIOESTE
}

\begin{abstract}
Resumo
Introdução: a simulação realística de alta fidelidade é uma metodologia empregada na área da saúde para formação profissional e carecem estudos que investigam a formação docente para o emprego no ensino superior. Objetivo: a pesquisa verificou a percepção docente após treinamento de docentes da área da saúde no emprego da simulação realística de alta fidelidade. Metodologia: tratase de uma abordagem qualitativa, cuja amostragem foi composta por onze docentes da área da saúde. Os sujeitos participaram de um treinamento em simulação realística de alta fidelidade envolvendo definição, objetivos, recursos, etapas metodológicas e operacionalização, aos quais, responderam dois questionários semiestruturados pré e pós-treinamento envolvendo o domínio de ensino e aprendizagem. Os dados colhidos foram submetidos à análise de conteúdo, do tipo temática, proposta por Bardin. Resultados: verificou-se que a concepção dos participantes acerca da simulação realística traz benefícios à prática docente, aproxima o discente da realidade profissional, sendo uma ferramenta útil no processo de ensino e aprendizagem, o domínio de novas tecnologias, além de estimular o desenvolvimento de habilidades e competências. Conclusão: os docentes apontaram que a simulação difere no ensino tradicional, diminui o risco dos discentes frente as situações clínicas, desenvolvendo habilidade e competências de tomada de decisão, criatividade, iniciativa, liderança, organização e gerenciamento do tempo.

Palavras-chave: Ensino. Simulação. Educação superior. Docentes.
\end{abstract}

\begin{abstract}
Introduction: realistic high-fidelity simulation is a methodology used in health sciences for professional training and studies that investigate faculty training for employment in higher education are lacking. Objective: to verify the faculty perception after training health professors in the use of realistic high-fidelity simulation. Methodology: a qualitative approach, whose sample consisted of eleven professors in the health field. The subjects participated in a training in realistic high-fidelity simulation involving definition, objectives, resources, methodological steps and operationalization, to which they answered two semi-structured questionnaires, preand post-training involving the teaching and learning domain. The data were submitted to content analysis, thematic type, proposed by Bardin. Results: the participants' conception about realistic simulation brings benefits to teaching practice, brings students closer to professional reality, being a useful tool in the teaching and learning process, the mastery of new technologies, in addition to stimulating development skills and competencies. Conclusion: Faculties pointed out that simulation differs from traditional teaching, decreases the risk of students in the face of clinical situations, developing skills and competencies for decision making, creativity, initiative, leadership, organization and time management.

Keywords: Teaching. Simulation. Higher education. Faculty.
\end{abstract}

\section{INTRODUÇÃO}

O Brasil cresce a passos curtos no cenário da educação, que cada vez mais, caminha para um modelo híbrido para o ensino superior, onde as instituições de ensino vêm mudando os currículos e a forma de ensinar seus alunos, haja vista a mudança do próprio perfil desses alunos, cada vez mais imersos em ambientes digitais, acostumados com acesso, interação e retenção de informações por diferentes mídias, adquirindo conhecimentos diversos, ainda que de maneira não formal.

Correspondente/Corresponding: *Marcelo Taglietti-End: Rua Curitiba 1591/304. CEP: 85802-227, Cascavel, Paraná, - Tel: (45) 3229-1558-

E-mail: marcelotaglietti@gmail.com.
Neste contexto, a introdução de tecnologias digitais tem presença cada vez mais marcante no ensino, por meio de diversos tipos de recursos disponíveis, o que favorece e amplia as diferentes formas de ensino (BARBOZA, 2016). Agregado às tecnologias digitais, o emprego de diferentes metodologias tem aparecido, retirando o foco do ensino tradicional muito centrado em aulas expositivas dialogadas para modelos onde os estudantes se tornam protagonistas, em ambientes de colaboração, mobilidade tecnológica, conexão contínua, engajamento e diversão, permitindo situações de ensino e aprendizagem inovadoras.

Dentre essas metodologias, Filatro e Cavalcanti (2018) apresentam um grupo de metodologias que se apoiam intensamente em mídias e tecnologias digitais, chamado de 
imersivas. Nesse grupo, o emprego das tecnologias digitais em educação deve ir além do quadro-negro, livros, lápis, canetas, cadernos, máquinas de projeção, lousas digitais, computadores, tabletes e outros artefatos. Para Kensky (2012), as tecnologias envolvem processos e produtos relacionados aos conhecimentos advindos da eletrônica, da microeletrônica e das telecomunicações. Na metodologia imersiva, o tipo de aprendizagem é baseado na imersão e na experiência, por exemplo, utilizando estratégias de gamificação, realidade virtual/aumentada, bem como o uso de diferentes tipos de simulação.

Dentre essas estratégias, a simulação é amplamente utilizada na área da Saúde para educação profissional e avaliação de profissionais (HEGLAND et al., 2017; ISSENBERG et al., 2005; LA CERRA et al., 2019). As simulações incluem dispositivos, pessoas treinadas, ambientes virtuais realistas e situações clínicas que imitam problemas, eventos ou condições que surgem em encontros profissionais (MOTOLA et al., 2013), e os simuladores utilizados na Saúde podem ser classificados como de baixa, moderada ou de alta fidelidade, de acordo com sua potencialidade de reproduzir precisamente imagens ou sons.

Os simuladores de baixa fidelidade são caracterizados como os simuladores estáticos, menos realísticos e usados para procedimentos específicos, a exemplo dos manequins de espuma usados para a prática de reanimação cardiopulmonar. Os simuladores de moderada fidelidade são mais realísticos, pois podem oferecer ausculta de sons respiratórios, cardíacos, pulsação, ou a identificação de diferentes diagnósticos. Os simuladores de alta fidelidade são manequins extremamente realísticos, pois muitos possuem movimentação torácica, olhos funcionais que piscam e reagem com a luz, sons cardíacos, pulmonares, gastrointestinais e vocais, apresentam sangramentos e secreções e reagem de acordo com as intervenções realizados pelos estudantes (MARAN; GLAVIN, 2003; SEROPIAN et al., 2004).

Os estudos que envolvem o uso simulação realística de alta fidelidade (SRAF) na área acadêmica são sólidos e permitem aos estudantes o desenvolvimento de habilidades e competências clínicas, possibilitando a análise crítica dos casos clínicos, o trabalho em equipe e a tomada de decisão (ABREU et al., 2014; LABRAGUE et al., 2019; YUAN et al., 2012). No entanto, uma revisão integrativa com vinte e cinco trabalhos brasileiros sobre o ensino baseado em simulação desenvolvido por Yamane et al., (2019) concluiu que são escassos os estudos que envolvam a atenção primária e aqueles que vão além das graduações de Medicina e Enfermagem.

Também é incipiente o número de artigos publicados que envolvam a percepção e a formação docente frente $o$ emprego de novas tecnologias educacionais como a SRAF. No âmbito nacional, encontramos apenas três pesquisas que investigaram a percepção docente frente ao uso da simulação realística de alta fidelidade.

A primeira, proposta por Gomez et al. (2010), analisaram o perfil de professores da área da Saúde que usam a simulação como estratégia didática. Para isso utilizaram a estratégia Delphi que reúne os consensos entre expertos no tema pesquisado, sendo entrevistados treze docentes do curso de Medicina, envolvendo trinte e dois quesitos, de cunho individual e social/profissional (identificação dos participantes: sexo; idade; formação; situação profissional; características das simulações e competências do professor). Os entrevistados concluíram que a simulação melhora a docência e o ensino, pois contribui com o trabalho em sala de aula, facilita a aprendizagem dos alunos e promove o acesso às novas tecnologias.

Já a segunda, com discentes e seis docentes do curso de Enfermagem, proposta por Espadaro (2017), objetivou o emprego da SRAF na percepção docente pelo emprego de um questionário semiestruturado envolvendo dez perguntas acerca do emprego da simulação no ensino da Enfermagem. Entre as temáticas, evidenciam-se a experiência utilizada na formação da graduação em Enfermagem, o planejamento para realização das atividades, a simulação como estratégia efetiva na formação do aluno, como desenvolvedora de habilidades e competências, possibilidade de curricularização da tecnologia e preparo dos docentes. Após esse questionário, duas docentes foram entrevistadas e foi realizada a análise qualitativa dos domínios da entrevista semiestruturada que envolveram temáticas de ensino e aprendizagem em simulação realística. Os entrevistados concluíram que a simulação realística influencia na qualidade da aprendizagem em Enfermagem provendo uma formação consolidada em padrões de segurança e eficiência.

A terceira, proposta por Carneiro et al. (2019) propôs identificar na perspectiva do corpo docente a importância da simulação realística na formação do profissional Enfermeiro. Por meio de uma abordagem quali-quantitativa descritiva, seis enfermeiras docentes responderam um questionário semiestruturado envolvendo as variáveis de ensino: experiência profissional em SRAF, frequência, uso, emprego e desenvolvimento de competências. Os pesquisadores concluíram que a SRAF apresenta vantagens relacionadas ao ensino, destacando a relação entre a teoria e a prática, o desenvolvimento de raciocínio clínico em casos complexos e individualizados e como desvantagem, a falta de capacitação do professor aliada a falta de condições de trabalho.

Esses trabalhos envolveram professores com títulos de pós-graduação e com experiência em SRAF, os quais as aplicam em seus currículos de graduação, que em sua maioria, também trabalha na área assistencial, o que favorece a criação de cenários para o uso da SRAF. Porém, quando se investigam trabalhos que analisam a formação docente no emprego da SRAF, não foram encontrados estudos. Com isso, entende-se ser esse um diferencial do presente artigo, por investigar essa formação com docentes do ensino superior da área da Saúde, bem como pelo fato de os participantes não terem tido contato prévio com a tecnologia.

Diante desse cenário, existe a necessidade de propiciar e relatar a formação docente para aplicação dessa 
tecnologia como ferramenta metodológica, na qual o professor promove toda a estrutura necessária para que o processo de ensino e aprendizagem ocorra, auxiliando os acadêmicos durante sua experiência com a simulação, bem como discutir os aspectos metodológicos, as percepções docentes e a operacionalização dos simuladores.

Por meio dessa hipótese, o objetivo então é investigar a percepção dos docentes da área da Saúde (Enfermagem e Fisioterapia) quanto a formação em simulação realística de alta fidelidade (inédita no brasil) e suas impressões após uma formação.

\section{METODOLOGIA}

Trata-se de um estudo qualitativo, desenvolvido no Laboratório de Altas Habilidades do Centro Universitário Assis Gurgacz (FAG), Cascavel, PR. O estudo seguiu as diretrizes para pesquisas com seres humanos, com aprovação pelo Comitê de Ética em Pesquisa mediante Parecer de número 25613619.6.0000.0107.

O treinamento em SRAF foi realizado durante a semana pedagógica da instituição de ensino superior (IES) supracitada realizada previamente ao início do ano letivo de 2020, onde os colegiados da área da Saúde divulgaram através de e-mail e mídia digital aos seus docentes a abertura de um workshop com no máximo vinte e cinco vagas. Dezoito docentes se inscreveram para o treinamento, porém, apenas onze docentes da área da Saúde participaram da capacitação teórico-prático em simulação realística de alta fidelidade, com duração de quatro horas. $O$ treinamento foi ministrado por três docentes da área da Saúde, pertencentes à IES, com experiência mínima de dois anos em SRAF, sendo duas enfermeiras mestres e um fisioterapeuta com título de doutor.

O treinamento envolveu definição, objetivos, recursos, etapas metodológicas da SRAF (preparo e aplicação) e, o componente prático envolveu a operacionalização dos dois bonecos, ambos adultos, um do gênero masculino (SimMan 3G - Laerdal Medical $^{\circledR}$ ) e outro do feminino (SimMom - Laerdal Medical ${ }^{\circledR}$ ) (Figura 1), e a realização de duas simulações: a de um caso clínico envolvendo parada cardiorrespiratória e a segunda, uma situação obstétrica de parto normal, descritas no Quadro 1.

Para a avaliação da percepção docente, dois questionários estruturados foram aplicados (antes e após treinamento). O primeiro, composto por dados pessoais envolvendo a formação docente, formação profissional, experiencia profissional e, questões abertas sobre o processo de ensino e aprendizagem frente ao uso da SRAF e as expectativas do treinamento. Já o segundo, investigou por meio de questões abertas e fechadas, as dimensões empregadas pela SRAF no processo de ensino e aprendizagem, o emprego da SRAF na prática docente, pontos positivos e negativos do uso da metodologia, bem como competências e habilidades docentes, finalizando com a avaliação do treinamento realizado.
Figura 1- Laboratório de altas habilidades e simuladores de alta fidelidade da IES.

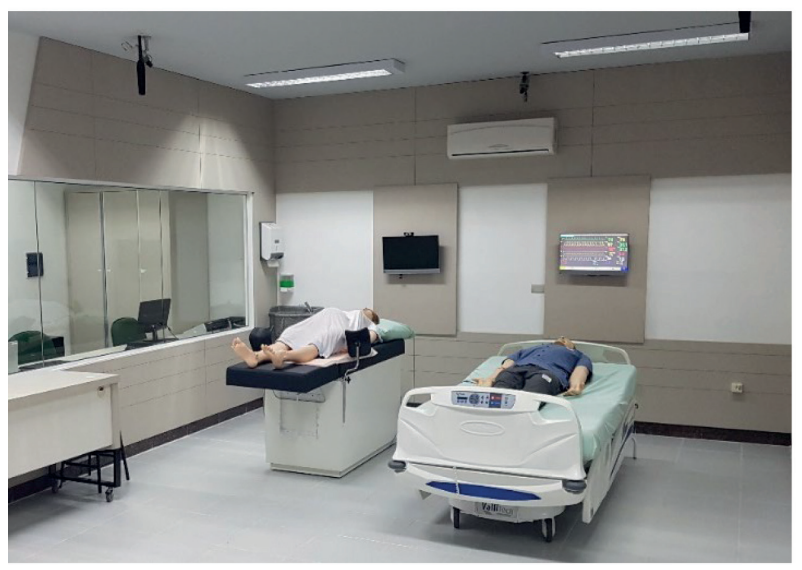

Fonte: Dados da pesquisa, 2021.

Quadro 1 - Sumário da sessão de formação docente em SRAF ofertada aos docentes.

\begin{tabular}{|l|l|}
\hline \multicolumn{2}{|l|}{ Formação docente em Simulação realística de alta fidelidade } \\
\hline $\mathbf{1}$ & $\begin{array}{l}\text { Objetivo: capacitar os docentes na área da Saúde no conhe- } \\
\text { cimento das etapas metodológicas da simulação e manuseio } \\
\text { da tecnologia. }\end{array}$ \\
\hline $\mathbf{2}$ & $\begin{array}{l}\text { Pessoal e equipamento: Instrutores, docentes da área da } \\
\text { Saúde, preparação do paciente, conhecimento do laboratório } \\
\text { e tecnologias. }\end{array}$ \\
\hline $\mathbf{3}$ & $\begin{array}{l}\text { Set up do computador e instruções operacionais: marcação dos } \\
\text { parâmetros fisiológicos dos cenários, estágios de simulação e } \\
\text { tratamentos esperados. }\end{array}$ \\
\hline $\mathbf{4}$ & $\begin{array}{l}\text { Documentações de suporte: prontuários dos pacientes, ECG, } \\
\text { Raio-x e exames complementares. }\end{array}$ \\
\hline $\mathbf{5}$ & $\begin{array}{l}\text { Contextualização: descrição da definição e fases da simulação } \\
\text { (fase de preparo e aplicação). }\end{array}$ \\
\hline $\mathbf{6}$ & $\begin{array}{l}\text { Descrição dos cenários (casos clínicos): descrição detalhadas } \\
\text { das duas situações propostas: parada cardiorrespiratória e } \\
\text { parto normal. }\end{array}$ \\
\hline $\mathbf{7}$ & $\begin{array}{l}\text { Aplicação: parte prática com resolução dos cenários pelos } \\
\text { docentes. }\end{array}$ \\
\hline $\mathbf{8}$ & $\begin{array}{l}\text { Debriefing: discussão sobre a aplicação prática, sugestões } \\
\text { para melhoramento dos cenários, soluções dos erros comuns } \\
\text { encontrados pelos participantes. }\end{array}$ \\
\hline
\end{tabular}

Fonte: Dados da pesquisa, 2021.

Para identificação nos discursos, foi utilizada a letra $P$, para participante, seguida da ordem de respostas dos questionários, de P1 a P11. Em relação a análise dos dados, os dados quantitativos foram apresentados em média e desvio-padrão e os dados qualitativos testados por meio de distribuição de frequência e apresentados em frequência e porcentagem.

As respostas às questões abertas foram submetidas à análise de conteúdo do tipo do conteúdo temático proposta por Bardin (2006), abrangendo as seguintes etapas: primeira com a realização da pré análise, na qual foram resumidas as ideias principais; segunda envolvendo a exploração das respostas, em que os dados foram transfor- 
mados em conteúdos temáticos, por meio da codificação dos discursos obtidos, determinando os temas a serem discutidos; e a terceira sendo a análise dos conteúdos, com a realização das inferências e interpretações dos resultados a partir dos objetivos que nortearam o estudo, cujo produto será apresentado em categorias, ilustradas com discursos dos docentes.

Foram identificadas as seguintes categorias analíticas para o questionário pré-workshop formativo: metodologia de ensino e aprendizagem clínica e expectativas na realização da oficina e; para o questionário pós-formação: dimensões no processo de ensino e aprendizagem; aplicabilidade na prática docente; limitações da SRAF; expectativas pós-treinamento e; formação docente no uso da SRAF.

\section{RESULTADOS E DISCUSSÃO}

Dos onze docentes, sete $(63,6 \%)$ eram do gênero feminino e quatro $(36,4 \%)$ do masculino, com média de idade de $36,7 \pm 6,4$ anos. Em relação a formação acadêmica seis $(54,5 \%)$ eram enfermeiros e cinco $(45,5 \%)$ fisioterapeutas, com escolaridade predominante de mestres com seis $(54,5 \%)$ docentes, especialistas quatro $(36,4 \%)$ e um $(9,1 \%)$ doutor. Todavia, quando investigada a experiência profissional, os participantes apresentaram média de $13,4 \pm 5,3$ anos de experiência e, em relação à experiência docente, a média apresentada foi de 9,5 5,8 anos no ensino superior.

Para o questionário pré-treinamento, três perguntas abertas geraram as categorias de metodologia de ensino e aprendizagem clínica e expectativas na realização da oficina, descritas a seguir.

Metodologia de ensino e aprendizagem clínica

Os docentes concluíram que a tecnologia é uma ferramenta que interfere no aprendizado do aluno e os principais argumentos relatados foram que ela simula situações reais sem oferecer riscos aos pacientes, modelo que difere do ensino tradicional, permite a aproximação da prática clínica e, possibilita situações clínicas básicas e complexas com alta fidelidade.

Essa metodologia é uma forma de simular situações reais sem oferecer riscos aos pacientes (P10).

Ela interfere positivamente no aprendizado do aluno, de forma que se sinta estimulado em ter aulas diferenciadas, diferentes das tradicionais (P8).

É uma didática de ensino excelente, devido poder expor situações que 0 aluno vivenciará tanto em campo de estágio, como na vida real e profissional (P2).

Aprender com a simulação é um caminho que leva a aprendizagem em sala de aula a experiência clínica real. Na presente pesquisa os docentes participaram de situações simples do dia a dia do profissional da Saúde como a aferição de sinais vitais, bem como a resolução de atividades complexas como um parto e uma parada cardiorrespiratória.
Espadaro (2017) afirma que as simulações de treinamentos podem diminuir a tensão, pois todos sabem que estão em uma realidade virtual, que os participantes não colocarão em risco os pacientes. Ela evita determinados perigos de configurações clínicas como instrumentos infectados, uso de equipamentos elétricos e fatores intervenientes que não são percebidos ou previstos na abordagem inicial do paciente. Oitenta e três por cento em sua pesquisa também concordaram que a SRAF é uma estratégia efetiva na formação dos alunos.

Filho e Scarpelini (2007) também apontam que SRAF na área da Saúde apresenta vantagens comparativas em relação a atuação numa situação real com a redução do risco para pacientes, relação de custo-benefício otimizada, método de aprendizado realizado por processos, estabelecimento de uma cultura de espírito de equipe e colaboração, a substituição de situações raras e o treinamento de competências cirúrgicas.

Além desses argumentos, Carneiro et al. (2019) complementam que a SRAF favorece a tomada de decisão do estudante junto ao seu treinamento, preparando-o para situações atípicas da prática clínica, melhora a comunicação e coordenação dos movimentos gerando precisão no manuseio de materiais bem como na execução de procedimentos.

O emprego da SRAF realmente se consolida como uma metodologia apropriada de ensino e aprendizagem clínica, haja vista seu potencial evidenciado nas pesquisas supracitadas e de fato, também pôde ser vista já no primeiro contato com os docentes que receberam a formação. É um recurso que favorece o aprendizado, em ambiente clínico seguro pré-estabelecido, que propicia o desenvolvimento de habilidades e competências.

\section{Expectativas na realização da oficina}

Os docentes almejam aprender o manuseio dos simuladores e das tecnologias, bem como aprender uma nova metodologia inovativa para o uso docente.

Expectativa de aprender uma nova metodologia para as aulas práticas, o manuseio de bonecos e aperfeiçoamento como docente (P5).

Desenvolver uma simulação frequentemente envolve um processo complexo que segue um conjunto de regras, relações e procedimentos operacionais, bem como o conhecimento de softwares e o manuseio de robôs. Para Gomez et al. (2011), a formação docente em habilidades e competências técnico-profissionais com base nos processos de simulação deve exigir uma atitude de aceitação das inovações pedagógicas por parte do docente em incorporar a estratégia de simulação, assim como estar aberto para revisar a sua metodologia de ensino e seu conceito do que seja educar na sociedade contemporânea.

Quando se avalia o questionário pós-treinamento, as respostas das perguntas abertas foram agrupadas nas 
seguintes categorias: dimensões no processo de ensino e aprendizagem, aplicabilidade na prática docente, pontos negativos da SRAF, expectativas pós-treinamento e, formação docente no uso da SRAF.

\section{Dimensões no processo de ensino e aprendizagem}

Felder e Soloman (1991) propõem quadro dimensões/estilos de aprendizagem: Ativo ou Reflexivo, Sensorial ou Intuitivo, Visual ou Verbal e Sequencial ou Global. Os participantes acreditam que o estilo Sequencial seja o mais desenvolvido com $5(45,5 \%)$ respostas, seguido do Ativo com $3(27,3 \%)$, Intuitivo $2(18,2 \%)$ e com apenas uma resposta $(9,1 \%)$, o estilo Visual.

O estilo Sequencial/Global indica a forma de organização do pensamento usado pelo estudante no caminho para o aprendizado. Os aprendizes globais necessitam de uma visão geral do conteúdo para poder assimilá-lo e, após isso, conseguirão relacionar os fragmentos descontextualizados da informação e, os aprendizes sequenciais, compreendem melhor através de uma sequência lógica, juntando as peças e assim construindo a visão geral sobre o assunto aprendido (VIDAL; LOMÔNACO, 2018).

A SRAF proporciona um ambiente controlado, onde os cenários são previamente explicados e, em seguida, são sequenciados e necessitam da finalização de cada etapa para posterior continuidade das atividades. Da mesma maneira, os discentes necessitam de uma postura pró ativa somada a tomada de decisão para resolver os problemas de cada etapa da simulação. Com isso, a simulação permite o desenvolvimento de todos os estilos de aprendizagem.

Identificar os estilos de aprendizagem dos discentes e propor estratégias de ensino neles baseados, devem estar presentes na formação dos docentes que irão trabalhar com SRAF. A sua identificação proporcionará a criação de cenários clínicos que melhor se adaptem as características dos participantes, favorecendo o aprendizado, bem como o desenvolvimento das habilidades e competências inerentes à prática profissional.

\section{Aplicabilidade na prática docente}

Todos os participantes concordaram que a metodologia é aplicável na prática de suas disciplinas, pois aproxima a realidade da prática clínica por meio de um ambiente seguro, possibilitando feedback instantâneo ao discente e, desenvolvendo habilidades e competências.

\section{Ótima oportunidade para criar situações problemas} em um ambiente seguro (P5).

No trabalho de Gomez et al. (2010) os docentes de Medicina também apontaram que a interação grupal é um fator forte da SRAF, bem como a metodologia a ser empregada, o interesse e pertinência dos temas, a formação dos formadores, a qualidade da simulação e a organização das atividades.

\section{Limitações da SRAF}

Como pontos negativos a necessidade de capacitação docente no manuseio dos bonecos e da tecnologia, e, resistência dos alunos frente às novas tecnologias foram apontadas pelos participantes.

Apresenta pontos positivos pois simula situações em ambiente seguro, porém exige muita capacitação para manipular os bonecos (P3).

Curioso foi o apontamento pelos docentes à resistência dos discentes pela imersão em novas tecnologias (SRAF). Na prática clínica dos ministrantes da formação e de um dos autores desse artigo, o que ocorre é exatamente o contrário. Os discentes se apresentam flexíveis, ativos, abertos e entusiasmados frente a essa possibilidade. A combinação da aprendizagem ativa com o uso de tecnologias digitais tem despertado cada vez mais o interesse e a participação dos acadêmicos.

Além desses argumentos, Gomez et al. (2010) também apontaram como desafios para a didática docente a integração da simulação aos conteúdos curriculares, manter a disciplina e a regularidade de uso e participação em processos de simulação, o tempo necessário para o desenvolvimento de novos cenários, tempo para corrigir e avaliar as atividades, e a orientação sobre o cuidado com os simuladores.

Para Carneiro et al. (2019) há algumas desvantagens ao uso da SRAF, como a inabilidade do professor associada a dificuldade de criar situações reais para o desenvolvimento da prática docente, a falta de conhecimento em tecnologias digitais em educação, a necessidade de constante atualização prejudicada devido a demanda de trabalho e a falta de tempo para dedicação a pesquisa. Ainda mais, levantaram a possibilidade da mudança de comportamento do estudante frente a ambientes reais de trabalho como hospitais e instituições similares, bem como o não domínio teórico dos conteúdos curriculares. Outro ponto de destaque dos autores foi a falta de interesse dos docentes pela tecnologia, a falta de treinamento, bem como condições de trabalho desfavoráveis como número excessivo de alunos e falta de materiais para as práticas.

O emprego da SRAF requer investimento financeiro e profissional alto perante as instituições de ensino, bem como treinamento e capacitação de todos envolvidos no processo de ensino e aprendizagem. Além disso, requer professores que detenham conhecimento assistencial para a criação dos cenários somado a um ambiente de trabalho que faça jus ao emprego dessa prática.

\section{Expectativas pós-treinamento em SRAF}

Quanto à satisfação, $7(63,6 \%)$ se mostraram completamente satisfeitos e $4(36,4 \%)$ muito satisfeitos, não havendo respostas negativas. Relataram ainda, que deve ocorrer a continuidade da formação, abordando o manuseio individual da tecnologia, bem como a prática com os discentes, simulando um ambiente real de sala de aula. 


\section{Formação docente no uso da SRAF}

Para a realização da simulação clínica, é indubitável a formação dos docentes que vão trabalhar com ela, pois é necessário que tenham clareza conceitual na sua abordagem, a fim de orientar a estratégia na formação dos futuros profissionais.

Para isso, são necessárias competências e habilidades já listadas como premissas por Caires (2013) para o desenvolvimento de simulações, envolvendo a comunicação, flexibilidade, empatia, postura, persuasão, ação estratégica, visão global e percepção realística. E para tal desenvolvimento, a formação e a percepção docente devem ser desenvolvidas no contato inicial da metodologia.

Na presente pesquisa, os participantes afirmaram a necessidade de treinamento prévio frente a essa nova tecnologia (robótica) além do domínio do conteúdo teórico, e apontaram como habilidades e competências docentes a criatividade, a iniciativa, o poder de decisão, a liderança, a organização e o gerenciamento do tempo.

\section{A simulação desperta no aluno a tomada de decisão, concentração, iniciativa e liderança (P4).}

Gomez et al. (2010) também consideram que um bom professor deve ser um bom comunicador e transmissor de conhecimentos, mais preocupado com a aprendizagem do que em mostrar que domina o assunto, ser um facilitador da aprendizagem, ter conhecimento e experiência no que está ensinando, saber ouvir, ser seguro e confiante no tema que ensina, manter interação com os alunos, despertar e manter o interesse do aluno, ser responsável e comprometido, ter clareza e praticidade no ensino relacionado com a realidade social e saber avaliar de forma coerente conforme a proposta desenvolvida.

A experiência em SRAF vivenciada pelos docentes foi efetiva em identificar as habilidades e competências que devem ser desenvolvidas pelos discentes. Cabe em futuras pesquisas, o desenvolvimento de cenários pelos participantes que as contemplem, direcionadas aos componentes temáticos da Enfermagem e Fisioterapia e sua inserção em seus currículos.

\section{CONCLUSÃO}

Por se tratar de estudo com o diferencial pela formação ofertada a docentes da área de Enfermagem e Fisioterapia em primeiro contato com a SRAF, nota-se que os objetivos foram alcançados, onde os professores foram conscientes dos desafios tecnológicos para o uso da SRAF no ensino a Saúde e apontaram que a simulação é uma ferramenta que difere do modelo tradicional de ensino, trazendo menos riscos aos discentes frente a situações que se assemelham à prática clínica, bem como é capaz do desenvolvimento de competências e habilidades.

Esse trabalho aponta que a formação é factível de realização, atende as profissões da Saúde, sendo bem absorvida a tecnologia de simulação realística de alta fidelidade como ferramenta de ensino nos cursos de Enfermagem e fisioterapia. Também deslumbra a práxis docente nas esferas desses cursos de graduação e sua empregabilidade acerca das temáticas envolvidas nesses cursos.

Ademais, todos os participantes trouxeram a percepção que o tempo utilizado para manuseio dos simuladores foi insuficiente. A operacionalização requer conhecimento dos simuladores, dos processos de simulação, do desenvolvimento dos cenários e das atividades pedagógicas voltadas aos componentes curriculares. Por hora, os professores se puseram como protagonistas diante dos dois cenários propostos pelos pesquisadores, requerendo práticas que envolvam o domínio de todas as etapas do método de simulação realística pelos docentes, e isso, prediz carga horária superior a esta imposta nesse estudo.

Sugere-se para futuras pesquisas, que se preconize carga horária mínima de doze horas, contemplando quatros horas envolvendo os componentes teóricos, quatro horas para operacionalização dos simuladores e as horas finais para desenvolvimento e aplicabilidade dos cenários. Todos se mostraram confiantes a continuidade do aprendizado perante essa nova ferramenta de ensino e cem por cento satisfeitos ao término da formação.

Sobre os discursos dos participantes, alguns apontamentos merecem destaque como a possibilidade de adesão da tecnologia nos currículos da área da Saúde bem como a formação docente para tal e como deveriam ser implementadas dos diferentes semestres da curricularização das profissões. Também relataram que as instituições devem promover a capacitação dos docentes e sua atualização periódica, bem como de práticas interdisciplinares envolvendo todos os colegiados de cursos da área da Saúde, não apenas os de Enfermagem e Fisioterapia.

A literatura carece de trabalhos na formação docente e os estudos futuros devem envolver a formação em todos as fases que envolvem a simulação realística de alta fidelidade, com carga horária correspondente, bem como trabalhos que apresentem as práticas pedagógicas da formação docente em simulação realística, aliada a introdução dessa prática nos currículos de formação em Saúde.

\section{REFERÊNCIAS}

ABREU, A. G. et al. O uso da simulação realística como metodologia de ensino e aprendizagem para as equipes de Enfermagem de um hospital infanto-juvenil: relato de experiência. Ciênc. Saúde, Porto Alegre, v. 7, n. 3, p. 162-166, set./dez. 2014.

BARBOZA, T. P. Tecnologias digitais: desafios e perspectivas no ensino superior em Saúde. Percurso Acadêmico, Belo Horizonte, v. 6, n. 12, jul./dez. 2016.

BARDIN, L. Análise de conteúdo. 5 ed. Lisboa: Edições 70, 2006.

CAIRES, J. C. Competências e formação de gestores multiplicadores. 2013. Disponivel em: http://www.convibra.org/upload/paper/adm/ adm_1115.pdf. Acesso em: 14 fev. 2020.

CARNEIRO, K. K. C. et al. Realist simulation as instrument for teachinglearning process in nursing. REVISA, São Paulo, v. 8, n. 3, p. 273-284, July/Dec. 2019. 
ESPADARO, R. F. Simulação realística na formação em enfermagem: percepção de docentes e discentes. 2017. 115f. Dissertação (Mestrado em Educação) - Universidade Nove de Julho, São Paulo, 2017.

FELDER, R. M.; SOLOMAN, B. A. Index of learning style. 1991. Disponível em: http: //www.ncsu.edu/felder-public/ILSpage.html. Acesso em: fev. 2020.

FILATRO, A.; CAVALCANTI, C. C. Metodologias Inov-ativas na educação presencial, a distância e corporativa. São Paulo: Saraiva Educação, 2018.

FILHO, A. P.; SCARPELINI, S. Simulação: Definição. Medicina (Ribeirão Preto), Ribeirão Preto, v. 40, n. 2, p. 162-166, abr./jun. 2007.

GOMEZ, M. V. et al. Análise do Perfil de Professores da área da saúde que usam a simulação como estratégia didática. Rev. Bras. Educ. Méd., Rio de Janeiro, v. 35, n. 2, p. 157-162, abr./jun. 2011.

HEGLAND, P. A. et al. Simulation-based training for nurses: systematic review and meta-analysis. Nurse Educ. Today, Edinburgh, v. 54, p. 6-20, Apr. 2017.

KENSKY, V. M. O que são tecnologias e por que elas são essenciais. In: KENSKY, Vani Moreira. Educação e tecnologias: o novo ritmo da informação. 8. ed. Campinas: Papirus, 2012.

LABRAGUE, L. J. et al. High-fidelity simulation and nursing students' anxiety and self-confidence: a systematic review. Nurs. Forum, Hillsdale, v. 54, n. 3, p. 358-368, July/Sept. 2019.

LA CERRA, C. et al. Effects of high-fidelity simulation based on life- threatening clinical condition scenarios on learning outcomes of undergraduate and postgraduate nursing students: a systematic review and meta-analysis. BMJ Open, Bethesda, v. 9, n. 2, p. 1-11, Feb. 2019.

MARAN, N. J.; GLAVIN, R. J. Low- to high-fidelity simulation-a continuum of medical education? Med. Educ. Edinburgh, v. 37, n.1, p. 22-28, Oct./ Dec. 2003.

MOTOLA, I. et al. Simulation in healthcare education: a best evidence practical guide. J. Med. Teacher, Miami, v. 35, p. 1-20, 2013.

SEROPIAN, M. A. et al. Simulation: not just a manikin. J. Nurs. Educ., Bethesda, v. 43, n. 4, p. 164-169, Apr. 2004.

VIDAL, L; LOMÔNACO, J. F. B. Estilos e estratégias de aprendizagem. [s.I]: Clube de Autores, 2018.

YAMANE, M. T. et al. Simulação realística como ferramenta de ensino na saúde: uma revisão integrativa. Rev. Espaço para a Saúde. Curitiba, v. 20, n. 1, p. 87-107, jul. 2019.

YUAN, H. B. et al. A systematic review of selected evidence on improving knowledge and skills through high-fidelity simulation. Nurse Educ. Today, v. 32, n. 3, p. 294-298, Apr. 2012.

Submetido em: 25/09/2020

Aceito em: 25/02/2021 